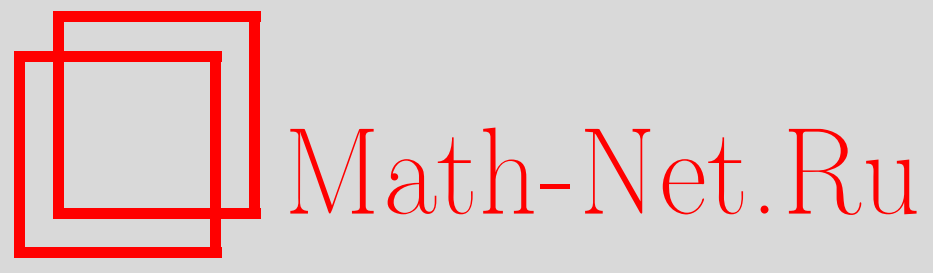

Ф. Г. Хуштова, Первая краевая задача в прямоугольной области для дифференциального уравнения с оператором Бесселя и частной производной Римана-Лиувилля, Вестн. Сам. гос. техн. ун-та. Сер. Физ.-мат. науки, 2021, номер 2, 241-256

DOI: https://doi.org/10.14498/vsgtu1820

Использование Общероссийского математического портала MathNet.Ru подразумевает, что вы прочитали и согласны с пользовательским соглашением

http: //www . mathnet.ru/rus/agreement

Параметры загрузки:

IP : 54.197 .217 .227

26 апреля 2023 г., $07: 21: 33$

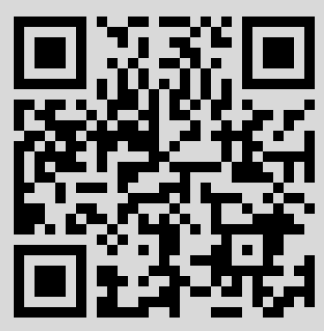


Вестн. Сам. гос. техн. ун-та. Сер. Физ.-мат. науки. 2021. Т. 25, № 2 . С. $241-256$ ISSN: 2310-7081 (online), 1991-8615 (print)

УДК 517.95

\title{
Первая краевая задача в прямоугольной области для дифференциального уравнения с оператором Бесселя и частной производной Римана-Лиувилля
}

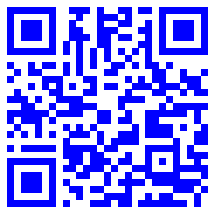

\section{(C) Ф. Г. Хуштова}

Институт прикладной математики и автоматизации КБНЦ РАН, Россия, 360000, Нальчик, ул. Шортанова, 89 а.

\begin{abstract}
Аннотация
Для дифференциального уравнения с сингулярным оператором Бесселя, действующим по пространственной переменной, и оператором дробного дифференцирования Римана-Лиувилля, действующим по временной переменной, рассматривается краевая задача в прямоугольной области с граничными условиями первого рода. Построено явное представление решения. Единственность решения доказана в классе функций, удовлетворяющих условию Гёльдера по временной переменной. Когда порядок дробной производной равен единице, а особенность у оператора Бесселя отсутствует, рассматриваемое уравнение совпадает с уравнением теплопроводности и полученные результаты совпадают с известными соответствующими классическими результатами.
\end{abstract}

Ключевые слова: уравнение дробной диффузии, оператор дробного дифференцирования, оператор Бесселя, цилиндрическая функция, функция типа Миттаг-Леффлера, первая краевая задача.

Получение: 18 августа 2020 г. / Исправление: 18 мая 2021 г. / Принятие: 24 мая 2021 г. / Публикация онлайн: 30 июня 2021 г.

\section{Научная статья}

(우 (7) Контент публикуется на условиях лицензии Creative Commons Attribution 4.0 International (https://creativecommons.org/licenses/by/4.0/deed.ru)

\section{Образец для цитирования}

Хуштова Ф. Г. Первая краевая задача в прямоугольной области для дифференциального уравнения с оператором Бесселя и частной производной Римана-Лиувилля // Вестн. Сам. гос. техн. ун-та. Сер. Физ.-мат. науки, 2021. Т. 25, № 2. С. 241-256. https://doi.org/10.14498/vsgtu1820.

\section{Сведения об авторе}

Фатима Гидовна Хуштова (1) https://orcid.org/0000-0003-4088-3621

кандидат физико-математических наук; научный сотрудник; отдел дробного исчисления; e-mail: khushtova@yandex.ru 


\section{Введение}

В области $\Omega=\{(x, y): 0<x<r, 0<y<T\}$ рассмотрим уравнение

$$
\mathbf{L} u \equiv B_{x} u(x, y)-D_{0 y}^{\alpha} u(x, y)=0,
$$

где $D_{0 y}^{\alpha}$ - оператор дробного дифференцирования в смысле Римана-Лиувилля порядка $0<\alpha \leqslant 1$, определяемый равенствами [1-3]: $D_{0 y}^{\alpha} g(y)=d g / d y$, если $\alpha=1$ и

$$
D_{0 y}^{\alpha} g(y)=\frac{1}{\Gamma(1-\alpha)} \frac{d}{d y} \int_{0}^{y} \frac{g(t)}{(y-t)^{\alpha}} d t
$$

если $0<\alpha<1$,

$$
B_{x}=x^{-b} \frac{\partial}{\partial x} x^{b} \frac{\partial}{\partial x}=\frac{\partial^{2}}{\partial x^{2}}+\frac{b}{x} \frac{\partial}{\partial x}
$$

- оператор Бесселя, $|b|<1$.

Частным случаем уравнения (1) является уравнение

$$
u_{x x}(x, y)+\frac{b}{x} u_{x}(x, y)-u_{y}(x, y)=0,
$$

которое в работе И. А. Киприянова $[4,5]$ носит название $B$-параболического уравнения. Дифференциальные уравнения с оператором Бесселя подробно и более полно исследованы в работах И. А. Киприянова и его учеников.

В работе Я. И. Житомирского [6] исследовалась краевая задача в первом квадранте для системы линейных уравнений в частных производных с дифференциальными операторами типа Бесселя

$$
\frac{\partial u(x, t)}{\partial t}=P(B, t) u(x, t)
$$

где $u(x, t)=\left\{u_{1}(x, t), \ldots, u_{m}(x, t)\right\} ; P(B, t)$ - квадратная матрица размера $m \times m$, элементами которой являются полиномы от операторов Бесселя

$$
B=\frac{\partial^{2}}{\partial x^{2}}+\frac{2 p+1}{x} \frac{\partial}{\partial x}
$$

одного и того же порядка $p \geqslant 0$.

Параболическим и эллиптическим уравнениям с оператором Бесселя посвящены также монографии $[7,8]$.

Различные краевые задачи для уравнения (1) в случае, когда оно совпадает с диффузионно-волновым уравнением, то есть $b=0,0<\alpha<2$, также богато и обстоятельно исследованы многими авторами. Подробную библиографию можно найти в работах [9-11].

Интерес к изучению уравнения (1) вызван его приложениями при моделировании процессов переноса во фрактальных средах [12-14].

Ранее задача Коши, первая и вторая краевые задачи в неограниченных областях для уравнения (1) были рассмотрены в работах [15-19]. 


\section{1. Постановка задачи}

Пусть $\widetilde{\Omega}=\Omega \cup\{y=T\}, \bar{\Omega}$ - замыкание области $\Omega$. Регулярным решением уравнения (1) в области $\Omega$ будем называть функцию $u=u(x, y)$, удовлетворяющую уравнению (1) в $\widetilde{\Omega}$ и такую, что $y^{1-\alpha} u \in C(\bar{\Omega}), B_{x} u, D_{0 y}^{\alpha} u \in C(\widetilde{\Omega})$.

ПЕРВАЯ КРАЕВАЯ ЗАДАЧА. Найти регулярное в области $\Omega$ решение уравнения (1), удовлетворяющее краевым условиям

$$
\begin{gathered}
\lim _{y \rightarrow 0} D_{0 y}^{\alpha-1} u(x, y)=\varphi(x), \quad 0 \leqslant x \leqslant r, \\
u(0, y)=u(r, y)=0, \quad 0 \leqslant y \leqslant T,
\end{gathered}
$$

где $\varphi(x)$ - заданная непрерывная функиия, $\varphi(0)=\varphi(r)=0$.

\section{2. Вспомогательные сведения}

2.1. Специальные функции. Далее в работе $J_{\nu}(z)$ - цилиндрическая функция Бесселя первого рода порядка $\nu$ [20, с. 132], [21, с. 95]; $I_{\nu}(z)$ и $K_{\nu}(z)-$ модифицированные цилиндрические функции Бесселя первого и второго рода порядка $\nu$ [20, с. 139], [21, с. 129,131$] ; E_{\rho, \mu}(z)$ - функция типа МиттагЛеффлера [22, с. 117].

Докажем следующее равенство:

$$
\frac{2^{2-\nu}}{\Gamma(\nu)} \sum_{m=1}^{\infty} \frac{J_{\nu}\left(\omega_{m} z\right)}{\omega_{m}^{2-\nu} J_{1+\nu}^{2}\left(\omega_{m}\right)}=z^{-\nu}-z^{\nu}, \quad 0<z \leqslant 1,
$$

где $\omega_{m}$ - положительные корни уравнения $J_{\nu}(\omega)=0$.

Разложим функцию

$$
f(z)=z^{-\nu}-z^{\nu}
$$

заданную на полуинтервале $(0 ; 1]$, в ряд Фурье--Бесселя [20, с. 163]

$$
f(z)=\sum_{m=1}^{\infty} c_{m} J_{\nu}\left(\omega_{m} z\right), \quad \nu>-1 / 2,
$$

где коэффициенты $c_{m}$ вычисляются по формуле

$$
c_{m}=\frac{2}{J_{1+\nu}^{2}\left(\omega_{m}\right)} \int_{0}^{1} z J_{\nu}\left(\omega_{m} z\right) f(z) d z, \quad m=1,2, \ldots
$$

Ряд (6) сходится к рассматриваемой функции (5), если $-1 / 2<\nu<3 / 2[20$, c. 165]. Подставим (5) в (7) и вычислим полученный интеграл с помощью формул (5.3.6) [20, с. 133]. Учитывая поведение функции $J_{\nu}(x)$ при $x \rightarrow 0[20$, c. 172], найдем

$$
\int_{0}^{1}\left[z^{1-\nu}-z^{1+\nu}\right] J_{\nu}\left(\omega_{m} z\right) d z=-\frac{1}{\omega_{m}}\left[J_{\nu-1}\left(\omega_{m}\right)+J_{\nu+1}\left(\omega_{m}\right)\right]+\frac{\omega_{m}^{\nu-2}}{2^{\nu-1} \Gamma(\nu)} .
$$

Тогда из (7), используя первую из формул (5.3.5) [20, с. 133] и условие

$$
J_{\nu}\left(\omega_{m}\right)=0, \quad m=1,2, \ldots,
$$


будем иметь

$$
c_{m}=\frac{2^{2-\nu}}{\Gamma(\nu)} \frac{\omega_{m}^{\nu-2}}{J_{1+\nu}^{2}\left(\omega_{m}\right)} .
$$

Подставляя найденные коэффициенты в (6), приходим к (4).

2.2. Интегральное преобразование Станковича. В работе Б. Станковича [24] рассмотрено обобщенное преобразование Ханкеля в виде

$$
G(x)=\int_{0}^{\infty} \phi\left(\delta, \mu+1 ;-x^{\delta} t\right) t^{\mu} g(t) d t, \quad \delta>0, \quad \mu>-1 .
$$

В работе А. В. Псху [25] (см. также [3, с. 72]) определено преобразование

$$
A^{\alpha, \mu} v(y)=y^{\mu-1} \int_{0}^{\infty} \phi\left(-\alpha, \mu ;-t y^{-\alpha}\right) v(t) d t, \quad 0<\alpha<1, \quad \mu \in \mathbb{R} .
$$

Достаточные условия сходимости интеграла (8) приведены в [3, с. 72]. Если $\mu=0$, то этот параметр опускается, то есть $A^{\alpha, 0} v(y)=A^{\alpha} v(y)$. Если функция $v$ зависит от нескольких переменных, то переменная, по которой проводится преобразование, обозначается в нижнем индексе, например, $A_{y}^{\alpha, \mu} v(x, y)$. В работе [26] исследованы различные формы обращения преобразования (8).

Пусть $0 \leqslant \mu \leqslant \alpha$ и $\lim _{y \rightarrow 0} D_{0 y}^{-\mu / \alpha} v(y)=v_{0}<\infty$. Тогда [3, с. 80]

$$
\lim _{y \rightarrow 0} D_{0 y}^{\alpha-1} A^{\alpha, \mu} v(y)=v_{0}
$$

Справедливы формулы [3, с. 84]

$$
\begin{gathered}
A^{\alpha} e^{\lambda y}=y^{\alpha-1} E_{\alpha, \alpha}\left(\lambda y^{\alpha}\right), \\
A^{\alpha / 2, \alpha / 2} \cos \lambda y=y^{\alpha-1} E_{\alpha, \alpha}\left(-\lambda^{2} y^{\alpha}\right) .
\end{gathered}
$$

\section{3. Основные результаты}

Обозначим $\beta=(1-b) / 2$,

$$
G(x, \xi, y)=\frac{2}{r^{2}} \frac{x^{\beta} \xi^{\beta}}{y^{1-\alpha}} \sum_{m=1}^{\infty} \frac{J_{\beta}\left(\lambda_{m} x\right) J_{\beta}\left(\lambda_{m} \xi\right)}{J_{1+\beta}^{2}\left(\lambda_{m} r\right)} E_{\alpha, \alpha}\left(-\lambda_{m}^{2} y^{\alpha}\right)
$$

где $\lambda_{m}$ - положительные корни уравнения $J_{\beta}\left(\lambda_{m} r\right)=0, m=1,2, \ldots$

ТЕОРема 1. Функиия

$$
u(x, y)=\int_{0}^{r} \xi^{1-2 \beta} G(x, \xi, y) \varphi(\xi) d \xi
$$

является решением задачи (1)-(3).

Доказательство. Докажем, что функция (13) удовлетворяет уравнению (1) и краевым условиям (2), (3). Для этого сначала покажем, что ряд 
(12) и ряды, которые получаются после применения к нему оператора Бесселя по $x$ и дробного дифференцирования по $y$ порядка $\alpha$, равномерно сходятся. Обозначим через

$$
X_{m}(x)=x^{\beta} J_{\beta}\left(\lambda_{m} x\right), \quad m=1,2, \ldots
$$

Функции $X_{m}(x)$ являются собственными функциями уравнения

$$
B_{x} X(x)+\lambda X(x)=0
$$

В силу ортогональности цилиндрических функций первого рода [21, с. 177] заключаем, что система функций (14) ортогональна с весом $x^{1-2 \beta}$ :

$$
\int_{0}^{r} x^{1-2 \beta} X_{m}(x) X_{l}(x) d x=\left\{\begin{array}{cl}
0, & m \neq l \\
\frac{r^{2}}{2} J_{\beta+1}^{2}\left(\lambda_{m} r\right), & m=l .
\end{array}\right.
$$

Для больших значений $\lambda$ справедливо неравенство [27, с. 274]

$$
\int_{0}^{\lambda} t J_{\beta}^{2}(t) d t \geqslant K \lambda, \quad K=\text { const }>0 .
$$

Из асимптотической формулы для функции $J_{\nu}(x)$ при $x \rightarrow \infty[20$, c. 172], (15) и последнего неравенства получим, что

$$
\frac{r^{2}}{2} J_{\beta+1}^{2}\left(\lambda_{m} r\right)=\int_{0}^{r} x J_{\beta}^{2}\left(\lambda_{m} x\right) d x=\frac{1}{\lambda_{m}^{2}} \int_{0}^{\lambda_{m} r} t J_{\beta}^{2}(t) d t \geqslant \frac{r K}{\lambda_{m}} .
$$

Тогда, учитывая асимптотические формулы (5.16.1) [20, с. 172] и (2.24) [22, с. 143] при больших значениях аргументов, для общего члена ряда (12) имеем

$$
G_{m}=O\left(\lambda_{m}^{-4}\right)
$$

Используя формулы (5.3.6) [20, с. 133], (1.2.12) [3, с. 15], а также (1.4) [22, с. 118], находим

$$
\begin{gathered}
B_{x} G(x, \xi, y)=-\frac{2}{r^{2}} \frac{x^{\beta} \xi^{\beta}}{y^{1-\alpha}} \sum_{m=1}^{\infty} \frac{\lambda_{m}^{2} J_{\beta}\left(\lambda_{m} x\right) J_{\beta}\left(\lambda_{m} \xi\right)}{J_{1+\beta}^{2}\left(\lambda_{m} r\right)} E_{\alpha, \alpha}\left(-\lambda_{m}^{2} y^{\alpha}\right), \\
D_{0 y}^{\alpha} G(x, \xi, y)=-\frac{2}{r^{2}} \frac{x^{\beta} \xi^{\beta}}{y^{1-\alpha}} \sum_{m=1}^{\infty} \frac{\lambda_{m}^{2} J_{\beta}\left(\lambda_{m} x\right) J_{\beta}\left(\lambda_{m} \xi\right)}{J_{1+\beta}^{2}\left(\lambda_{m} r\right)} E_{\alpha, \alpha}\left(-\lambda_{m}^{2} y^{\alpha}\right) .
\end{gathered}
$$

Общие члены этих рядов будут иметь оценки

$$
B_{x} G_{m}=O\left(\lambda_{m}^{-2}\right), \quad D_{0 y}^{\alpha} G_{m}=O\left(\lambda_{m}^{-2}\right) .
$$

Отсюда следует абсолютная и равномерная сходимость рядов (12), (16) и (17).

Подставляя (16) и (17) в уравнение (1), убеждаемся, что оно обращается в тождество. 
Чтобы доказать выполнимость условия (2), прежде покажем, что представление функции (12) эквивалентно представлению

$$
G(x, \xi, y)=A_{y}^{\alpha} g(x, \xi, y)
$$

где

$$
\begin{gathered}
g(x, \xi, y)=\frac{1}{2 \pi i} \int_{\gamma-i \infty}^{\gamma+i \infty} e^{y s} \widetilde{g}(x, \xi, s) d s, \quad \gamma>0, \\
\widetilde{g}(x, \xi, s)=\left\{\begin{array}{cc}
\Delta(\xi, x, s), \quad 0 \leqslant \xi \leqslant x, \\
\Delta(x, \xi, s), \quad x \leqslant \xi \leqslant r,
\end{array}\right. \\
\Delta(x, \xi, s)=x^{\beta} \xi^{\beta} \frac{I_{\beta}(\sqrt{s} x)}{I_{\beta}(\sqrt{s} r)}\left[I_{\beta}(\sqrt{s} r) K_{\beta}(\sqrt{s} \xi)-K_{\beta}(\sqrt{s} r) I_{\beta}(\sqrt{s} \xi)\right] .
\end{gathered}
$$

Определим порядок величины функции $\widetilde{g}(x, \xi, s)$ в интеграле (18). Пусть

$$
s=R e^{i \theta}, \quad 0 \leqslant \theta \leqslant \theta_{0}<\pi, \quad 0<\xi<x .
$$

Тогда

$$
\left|e^{\sqrt{s}(\xi-r)}\right|<e^{\sqrt{R}(\xi-r) \cos (\theta / 2)}, \quad|\operatorname{sh} \sqrt{s}(r-x)|<e^{\sqrt{R}(r-x) \cos (\theta / 2)} .
$$

Используя асимптотические формулы (5.16.5) для функций $I_{\nu}(x)$ и $K_{\nu}(x)$ при больших значениях аргумента [20, с. 173] из последних оценок находим

$$
|\widetilde{g}(x, \xi, s)|<\text { const } \cdot R^{-1 / 2} e^{-\sqrt{R}(x-\xi) \cos (\theta / 2)} .
$$

Так как функция (19) есть однозначная функция переменной $s$, для вычисления интеграла (18) будем использовать контур, представленный на рис. 1. Полюсами функции $\widetilde{g}(x, \xi, s)$ будут являться нули функции $I_{\beta}(\sqrt{s} r)$, то есть $s=-\lambda_{m}^{2}$, где $\pm \lambda_{m}$ - корни уравнения $J_{\beta}\left(\lambda_{m} r\right)=0, m=1,2, \ldots$ По теореме Коши [28] интеграл (18) есть произведение $2 \pi i$ на сумму вычетов относительно полюсов подынтегральной функции.

При $0 \leqslant \xi \leqslant x$ получаем

$$
g(x, \xi, y)=x^{\beta} \xi^{\beta} \sum_{m=1}^{\infty} \frac{I_{\beta}\left(i \lambda_{m} \xi\right)\left[-K_{\beta}\left(i \lambda_{m} r\right) I_{\beta}\left(i \lambda_{m} x\right)\right]}{\left[\frac{d}{d s} I_{\beta}(\sqrt{s} r)\right]_{s=-\lambda_{m}^{2}}} e^{-\lambda_{m}^{2} t}
$$

где суммирование ведется по положительным корням уравнения $J_{\beta}\left(\lambda_{m} r\right)=0$, занумерованным в порядке их возрастания.

Из равенств

$$
\frac{d}{d s} I_{\beta}(\sqrt{s} r)=\frac{r}{2 \sqrt{s}} I_{\beta}^{\prime}(\sqrt{s} r)
$$

и (5.9.5) [20, с. 145], а также формулы

$$
z I_{\beta}^{\prime}(z)-\beta I_{\beta}(z)=z I_{\beta+1}(z),
$$




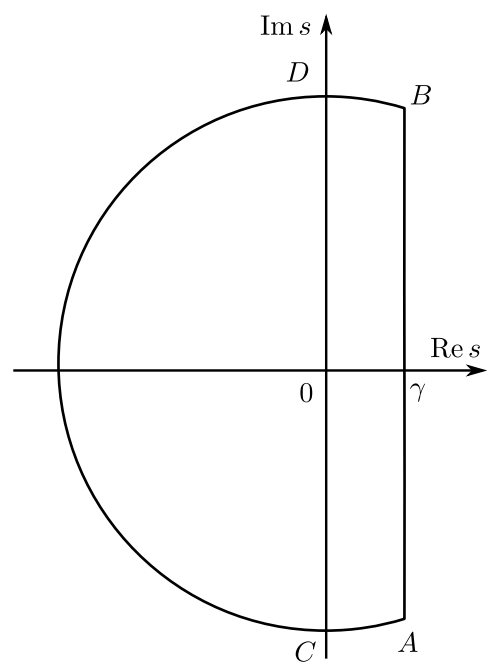

Рис. 1. [Figure 1]

которую легко можно вывести из формул (5.7.9) [20, с. 141], следует

$$
g(x, \xi, y)=\frac{2 x^{\beta} \xi^{\beta}}{r^{2}} \sum_{m=1}^{\infty} \frac{J_{\beta}\left(\lambda_{m} x\right) J_{\beta}\left(\lambda_{m} \xi\right)}{J_{1+\beta}^{2}\left(\lambda_{m} r\right)} e^{-\lambda_{m}^{2} y}
$$

$\mathrm{K}$ равенству (23) применим преобразование $A^{\alpha}$ по переменной $y$ по формуле $(10)$. В результате для функции $G(x, \xi, y)$ придем к представлению (12), симметричному относительно $x$ и $\xi$ и в силу последнего верному также при $x \leqslant \xi \leqslant r$.

Контур интегрирования $L=(\gamma-i \infty, \gamma+i \infty)$ в (18) при $y>0$ и $0<\xi<r$ может быть заменен контуром $L^{*}$, который начинается в бесконечно удаленной точке в направлении $\arg s=-\theta_{1}$, где $\pi / 2<\theta_{1}<\pi$, огибает начало координат справа, оставляя все особенности подынтегральной функции слева, и заканчивается в бесконечно удаленной точке в направлении $\arg s=\theta_{1}$ (рис. 2). Покажем, что интеграл

$$
\int e^{y s} \widetilde{g}(x, \xi, s) d s
$$

по дугам $B D F$ и $E C A$ окружности радиуса $R$ стремится к нулю, когда $R \rightarrow \infty$. Учитывая условия (21), можем записать

$$
0<e^{-\sqrt{R}(x-\xi) \cos (\theta / 2)}<1 .
$$

Обозначим через $I_{1}$ и $I_{2}$ интегралы вдоль дуг $B D$ и $D F$, а на дуге $B D$ положим $\omega=\arccos (\gamma / R)$. Тогда из (22) найдем

$$
\left|I_{1}\right|<\mathrm{const} \cdot \sqrt{R} e^{\gamma y} \int_{\omega}^{\pi / 2} d \theta=\mathrm{const} \cdot \sqrt{R} e^{\gamma y} \arcsin \frac{\gamma}{R} .
$$

Отсюда следует, что $\lim _{R \rightarrow \infty} I_{1}=0$. 


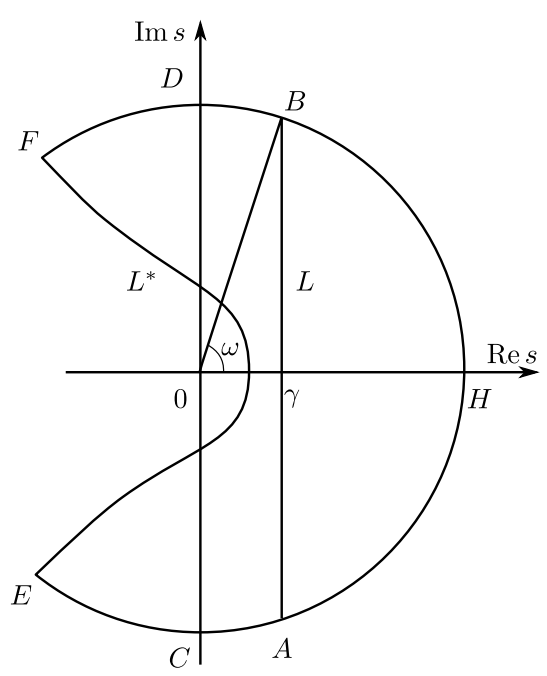

Рис. 2. [Figure 2]

Заменяя в $(21) \theta_{0}$ через $\theta_{1}>\pi / 2$ и принимая во внимание оценку $\sin \theta \geqslant$ $\geqslant 2 \theta / \pi$, справедливую при $0 \leqslant \theta \leqslant \pi / 2$, для интеграла $I_{2}$ получим

$$
\begin{array}{r}
\left|I_{2}\right|<\text { const } \cdot \sqrt{R} \int_{\pi / 2}^{\theta_{1}} e^{R t \cos \theta} d \theta<\text { const } \cdot \sqrt{R} \int_{0}^{\theta_{1}-\pi / 2} e^{-R t \sin \theta} d \theta< \\
<\text { const } \cdot \sqrt{R} \int_{0}^{\pi / 2} e^{-2 R t \theta / \pi} d \theta<\frac{\text { const }}{\sqrt{R} t},
\end{array}
$$

откуда $\lim _{R \rightarrow \infty} I_{2}=0$.

Аналогичные результаты можно получить и для интегралов по дугам $A C$ и $C E$. В силу вышеприведенных рассуждений функцию $g(x, \xi, y)$ можно записать следующим образом:

$$
g(x, \xi, y)=\frac{1}{2 \pi i} \int_{L^{*}} e^{t s} \widetilde{g}(x, \xi, s) d s,
$$

где $\widetilde{g}(x, \xi, s)$ определяется из (19).

Заметим, что функция $g(x, \xi, y)$ является непрерывной функцией от $y$ при $y \geqslant 0, x \neq \xi$, так как в силу оценки (22) при фиксированных $x \neq \xi$ интеграл (24) равномерно сходится при $y \geqslant 0$.

В силу формулы (9) можно записать:

$$
\begin{aligned}
\lim _{y \rightarrow 0} \int_{0}^{r} \xi^{1-2 \beta} D_{0 y}^{\alpha-1} G(x, \xi, y) d \xi= & \\
=\lim _{y \rightarrow 0}\left[\int_{0}^{r} \xi^{1-2 \beta} g(x, \xi, y)[\varphi(\xi)-\varphi(x)] d \xi+\varphi(x)\right. & \left.\int_{0}^{r} \xi^{1-2 \beta} g(x, \xi, y) d \xi\right]= \\
& =\lim _{y \rightarrow 0}\left[J_{1}(x, y)+J_{2}(x, y)\right] .
\end{aligned}
$$


Представим интеграл $J_{1}(x, y)$ в виде

$$
\begin{gathered}
J_{1}(x, y)=\int_{0}^{x-\varepsilon} \xi^{1-2 \beta} g(x, \xi, y)[\varphi(\xi)-\varphi(x)] d \xi+ \\
+\int_{x-\varepsilon}^{x+\varepsilon} \xi^{1-2 \beta} g(x, \xi, y)[\varphi(\xi)-\varphi(x)] d \xi+\int_{x+\varepsilon}^{r} \xi^{1-2 \beta} g(x, \xi, y)[\varphi(\xi)-\varphi(x)] d \xi= \\
=J_{11}(x, y)+J_{12}(x, y)+J_{13}(x, y),
\end{gathered}
$$

где $\varepsilon>0$.

Так как функция $g(x, \xi, y)$ непрерывна при $y \geqslant 0, x \neq \xi$, то

$$
\lim _{y \rightarrow 0} g(x, \xi, y)=\frac{1}{2 \pi i} \int_{L^{*}} \widetilde{g}(x, \xi, s) d s .
$$

Вдоль дуги $F D H A E$ имеет место оценка

$$
|\widetilde{g}(x, \xi, s)|<x^{\beta-1 / 2} \xi^{\beta-1 / 2} R^{-1 / 2} e^{-\sqrt{R}(x-\xi) \cos (\theta / 2)} .
$$

Отсюда следует, что интеграл

$$
\int \widetilde{g}(x, \xi, s) d s
$$

по этой дуге стремится к нулю при $R \rightarrow \infty$. Так как внутри замкнутого контура, изображенного на рис. 2 , нет полюсов, то имеем

$$
\int_{L^{*}} \widetilde{g}(x, \xi, s) d s=0 .
$$

Тогда $\lim _{y \rightarrow 0} J_{11}(x, y)=\lim _{y \rightarrow 0} J_{13}(x, y)=0$.

Пусть $\omega(\varepsilon)=\sup |\varphi(x)-\varphi(\xi)|, \xi \in[x-\varepsilon, x+\varepsilon]$. Так как функция $\varphi(x)$ непрерывна на отрезке $[x-\varepsilon, x+\varepsilon]$, то $\omega(\varepsilon) \rightarrow 0$ при $\varepsilon \rightarrow 0$. Из оценки $(25)$ и произвольности выбора $\varepsilon$ получаем $\lim _{y \rightarrow 0} J_{12}(x, y)=0$.

Для вычисления интеграла $J_{2}(x, y)$ воспользуемся представлением $(23)$. В силу второй из формул (5.3.6) [20, с. 133] поведения функции $J_{\beta-1}(x)$ при $x \rightarrow 0[20$, с. 172] и первой из формул (5.3.5) [20, с. 133] при $\nu=\beta$, а также учитывая, что $J_{\beta}\left(\lambda_{m} r\right)=0$, будем иметь

$$
\int_{0}^{r} \xi^{1-\beta} J_{\beta}\left(\lambda_{m} \xi\right) d \xi=\frac{r^{1-\beta}}{\lambda_{m}} J_{\beta+1}\left(\lambda_{m} r\right)+\frac{\lambda_{m}^{\beta-2}}{2^{\beta-1} \Gamma(\beta)} .
$$

Тогда при $y \rightarrow 0$ для $J_{2}(x, y)$ получим

$$
\begin{aligned}
\lim _{y \rightarrow 0} J_{2}(x, y)=\frac{2 x^{\beta} \varphi(x)}{r^{1+\beta} \Gamma(\alpha)} \sum_{m=1}^{\infty} \frac{J_{\beta}\left(\lambda_{m} x\right)}{\lambda_{m} J_{1+\beta}\left(\lambda_{m} r\right)}+ & \\
& +\frac{x^{\beta} \varphi(x)}{r^{2} \Gamma(\alpha)} \frac{2^{2-\beta}}{\Gamma(\beta)} \sum_{m=1}^{\infty} \frac{J_{\beta}\left(\lambda_{m} x\right)}{\lambda_{m}^{2-\beta} J_{1+\beta}^{2}\left(\lambda_{m} r\right)} .
\end{aligned}
$$


Отсюда, в силу равенств 5.7 .33 (1) [23, с. 610] и (4), окончательно получим $\lim _{y \rightarrow 0} J_{2}(x, y)=\varphi(x)$.

Теорема 2. Существует не более одного регулярного решения задачи (1)(3), удовлетворяющего для любого $x \in(0, r)$ условию

$$
|u(x, y)-u(x, t)| \leqslant k(y-t)^{\lambda}, \quad k=k(x)>0, \lambda>\alpha, \forall y \geqslant t>0 .
$$

Доказ ательст в о. Пусть $u(x, y)$ - решение однородной задачи

$$
\begin{gathered}
\mathbf{L} u \equiv 0 \text { при }(x, y) \in \widetilde{\Omega}, \\
u(0, y)=u(r, y)=0, \quad 0<y<T \text { и } \lim _{y \rightarrow 0} D_{0 y}^{\alpha-1} u(x, y)=0, \quad 0 \leqslant x \leqslant r .
\end{gathered}
$$

Последнее равенство равносильно тому, что

$$
\lim _{y \rightarrow 0} y^{1-\alpha} u(x, y)=0, \quad 0 \leqslant x \leqslant r .
$$

Необходимо показать, что $u(x, y) \equiv 0$ на $\widetilde{\Omega}$. Допустим, что это не так, то есть существует точка $(\xi, \eta) \in \widetilde{\Omega}$ такая, что $u(\xi, \eta) \neq 0$. Не теряя общности, будем считать, что $u(\xi, \eta)>0$. Тогда

$$
\max _{\bar{\Omega}} y^{1-\alpha} u(x, y)=\eta^{1-\alpha} u(\xi, \eta)>0, \quad(\xi, \eta) \in \widetilde{\Omega} .
$$

В этой точке в силу необходимых условий экстремума имеем

$$
u_{x}(\xi, \eta)=0, \quad u_{x x}(\xi, \eta) \leqslant 0 .
$$

С другой стороны, в силу условий (26) и (27) из принципа экстремума для оператора дробного дифференцирования следует [29]

$$
\left.D_{0 y}^{\alpha} u(\xi, y)\right|_{y=\eta} \geqslant 0
$$

причем равенство в последнем неравенстве возможно только в случае, когда $y^{1-\alpha} u(x, y)=$ const. В последнем случае сразу же заключаем, что $u(x, y) \equiv 0$ на $\widetilde{\Omega}$. Если $y^{1-\alpha} u(x, y) \neq$ const, то $\mathbf{L} u<0$ в точке $(\xi, \eta)$. Последнее неравенство противоречит условию $\mathbf{L} u(\xi, \eta)=0$. Следовательно, наше допущение неверно, поэтому $u(x, y) \equiv 0$ на $\widetilde{\Omega}$.

\section{4. Неоднородные граничные условия и частный случай}

Решение первой краевой задачи для уравнения (1) в случае, когда $\varphi(x) \equiv 0$ И

$$
u(0, y)=\tau_{0}(y), \quad u(r, y)=\tau_{r}(y), \quad 0<y<T,
$$

может быть представлено в виде

$$
u(x, y)=\left.\int_{0}^{y} \xi^{1-2 \beta} G_{\xi}(x, \xi, y-\eta)\right|_{\xi=0} \tau_{0}(\eta) d \eta-r^{1-2 \beta} \int_{0}^{y} G_{\xi}(x, r, y-\eta) \tau_{r}(\eta) d \eta .
$$


При этом от заданных функций $\tau_{0}(y)$ и $\tau_{r}(y)$ нужно требовать выполнения условий $y^{1-\alpha} \tau_{0}(y), y^{1-\alpha} \tau_{r}(y) \in C[0, T], \lim _{y \rightarrow 0} D_{0 y}^{\alpha-1} \tau_{0}(y)=\lim _{y \rightarrow 0} D_{0 y}^{\alpha-1} \tau_{r}(y)=0$.

Заметим, что в силу первого представления (1.2) [22, с. 118] функция (12) при $\alpha=1$ примет вид

$$
G(x, \xi, y)=\frac{2 x^{\beta} \xi^{\beta}}{r^{2}} \sum_{m=1}^{\infty} \frac{J_{\beta}\left(\lambda_{m} x\right) J_{\beta}\left(\lambda_{m} \xi\right)}{J_{1+\beta}^{2}\left(\lambda_{m} r\right)} e^{-\lambda_{m}^{2} y}
$$

а при $\beta=1 / 2$ из (5.8.1) и (5.8.2) [20, с. 142] следует

$$
G(x, \xi, y)=\frac{2 y^{\alpha-1}}{r} \sum_{m=1}^{\infty} \sin \frac{m \pi x}{r} \sin \frac{m \pi \xi}{r} E_{\alpha, \alpha}\left(-\left(\frac{m \pi}{r}\right)^{2} y^{\alpha}\right)
$$

В свою очередь, из (28) при $\beta=1 / 2$ и (29) при $\alpha=1$ получаем функцию

$$
G(x, \xi, y)=\frac{2}{r} \sum_{m=1}^{\infty} \sin \frac{m \pi x}{r} \sin \frac{m \pi \xi}{r} \exp \left(-\left(\frac{m \pi}{r}\right)^{2} y\right)
$$

которая совпадает с функцией Грина (или функцией мгновенного точечного источника тепла) первой краевой задачи в прямоугольной области для уравнения теплопроводности [30, с. 205], [31, с. 472]. Различные краевые задачи для уравнения теплопроводности, записанного в цилиндрических и сферических координатах, можно найти в работах [30, с. 464], [31, с. 473].

В случае, когда $\beta=1 / 2(b=0)$, решение первой краевой задачи в прямоугольной области для уравнения диффузии дробного порядка в терминах функции Райта приведено в работе [3, с. 108]. Эквивалентность представления из этой работы и представления (29) нетрудно показать, используя формулу (11) и проведя рассуждения, аналогичные приведенным в [30, с. 475].

\section{5. Случай $r=\infty$}

Устремляя $r$ к бесконечности в (20) и используя асимптотические формулы (5.16.5) [20, с. 173], получаем

$$
\Delta(x, \xi, s)=x^{\beta} \xi^{\beta} I_{\beta}(\sqrt{s} x) K_{\beta}(\sqrt{s} \xi)
$$

Тогда из формулы (116) [32, с. 590] следует, что функция

$$
G(x, \xi, y)=A_{y}^{\alpha} g(x, \xi, y), \quad g(x, \xi, y)=\frac{x^{\beta} \xi^{\beta}}{2 y} \exp \left(-\frac{x^{2}+\xi^{2}}{4 y}\right) I_{\beta}\left(\frac{x \xi}{2 y}\right)
$$

совпадает с функцией Грина первой краевой задачи для уравнения (1) в полуполосе [18]. 


\section{Заключение}

В данной работе рассматривается краевая задача в прямоугольной области с граничными условиями первого рода для дифференциального уравнения с оператором Бесселя и частной производной Римана-Лиувилля. В терминах специальных функций построено представление решения и доказана его единственность в классе функций, удовлетворяющих условию Гёльдера по временной переменной.

Конкурирующие интересы. Конкурирующих интересов не имею.

Авторская ответственность. Я несу полную ответственность за предоставление окончательной версии рукописи в печать. Окончательная версия рукописи мною одобрена.

Финансирование. Исследование выполнялось без финансирования.

Благодарности. Автор благодарит двух анонимных рецензентов за внимательное прочтение статьи, ценные предложения и комментарии.

\section{Библиографический список}

1. Самко С. Г., Килбас А. А., Маричев О. И. Интеграль и производные дробного порядка и некоторые их приложения. Минск: Наука и техника, 1987. 688 с.

2. Нахушев А. М. Дробное исчисление и его применение. М.: Физматлит, 2003. 272 с.

3. Псху А. В. Уравнения в частных производных дробного порядка. М.: Наука, 2005. 199 с.

4. Киприянов И. А., Катрахов В. В., Ляпин В. М. О краевых задачах в областях общего вида для сингулярных параболических систем уравнений// Докл. AH CCCP, 1976. Т. 230, №6. С. 1271-1274.

5. Киприянов И. А. Сингулярные эллиптические краевые задачи. М.: Наука, 1997. 204 с.

6. Житомирский Я. И. Задача Коши для систем линейных уравнений в частных производных с дифференциальными операторами типа Бесселя// Матем. сб., 1955. Т. 36(78), № 2. С. 299-310.

7. Матійчук М. І. Параболічні сингулярні крайові задачі. Київ: Ін-т математики НАН України, 1999. 176 с.

8. Матійчук М. І. Параболічні та еліптичні крайові задачі з особливостями. Чернівці: Прут, 2003. 284 с.

9. Псху А. В. Первая краевая задача для дробного диффузионно-волнового уравнения в нецилиндрической области // Изв. РАН. Сер. матем., 2017. Т. 81, №6. С. 158-179. https://doi.org/10.4213/im8520.

10. Podlubny I. Fractional Differential Equations. An introduction to fractional derivatives, fractional differential equations, to methods of their solution and some of their applications / Mathematics in Science and Engineering. vol. 198. San Diego, CA: Academic Press, 1999. xxiv +340 pp.

11. Kilbas A. A., Srivastava H. M., Trujillo J. J. Theory and Applications of Fractional Differential Equations / North-Holland Mathematics Studies. vol. 204. Amsterdam: Elsevier, 2006. xv+523 pp. https://doi.org/10.1016/S0304-0208(06)80001-0.

12. Metzler R., Glöckle W. G., Nonnenmacher T. F. Fractional model equation for anomalous diffusion// Physica A. Stat. Mech. Appl., 1994. vol.211. pp. 13-24. https://doi.org/10. 1016/0378-4371(94)90064-7.

13. Metzler R., Klafter J. The random walk's guide to anomalous diffusion: a fractional dynamics approach// Phys. Reports, 2000. vol.339, no.1. pp. 1-77. https://doi.org/ 10.1016/S0370-1573 (00) 00070-3.

14. Uchaikin V. V. Fractional Derivatives for Physicists and Engineers. vol. 1: Background and Theory. Berlin: Springer, 2013. xxi+385 pp. https://doi.org/10.1007/ 978-3-642-33911-0. 
15. Хуштова Ф. Г. Задача Коши для уравнения параболического типа с оператором Бесселя и частной производной Римана-Лиувилля // Вестн. Сам. гос. техн. ун-та. Сер. Физ.-мат. науки, 2016. Т. 20, №1. С. 74-84. https://doi.org/10.14498/vsgtu1455.

16. Хуштова Ф. Г. Первая краевая задача в полуполосе для уравнения параболического типа с оператором Бесселя и производной Римана-Лиувилля // Матем. заметки, 2016. T. 99, № 6. C. 921-928. https://doi.org/10.4213/mzm10759.

17. Хуштова Ф. Г. Вторая краевая задача в полуполосе для уравнения параболического типа с оператором Бесселя и частной производной Римана-Лиувилля // Maтем. заметки, 2018. T. 103, №3. C. 460-470. https://doi.org/10.4213/mzm10986.

18. Хуштова Ф. Г. Первая краевая задача в полуполосе для дробно-дифференциального уравнения с оператором Бесселя и частной производной Римана-Лиувилля // Уфимск. матем. журн., 2017. Т. 9, № 4. С. 117-128. https://doi.org/10.13108/2017-9-4-114.

19. Хуштова Ф. Г. Вторая краевая задача в полуполосе для уравнения параболического типа с оператором Бесселя и производной Римана-Лиувилля// Изв. вузов. Матем., 2017. № 7. C. 84-93.

20. Лебедев Н. Н. Специальные функиии и их приложения. М.: Физматлит, 1963. 359 с.

21. Кузнецов Д. С. Специальные функиии. М.: Высш. шк., 1962. 248 с.

22. Джрбашян М. М. Интегральные преобразования и представления функиий в комплексной области. М.: Наука, 1966. 672 с.

23. Прудников А. П., Брычков Ю. А., Маричев О. И. Интегралы и ряды. Т. 2: Специальные функции. М.: Физматлит, 2003. 664 с.

24. Stanković B. Inversion et invariantes de la transformation généralisée de Hankel// Acad. Serbe Sci., Publ. Inst. Math., 1955. vol. 8. pp. 37-52 (In French).

25. Псху А. В. Интегральное преобразование с функцией Райта в ядре// Докл. Адъгской (Черкесской) Междунар. Акад., 2002. Т. 6, №1. С. 35-47.

26. Псху А. В. Об обращении интегрального преобразования Б. Станковича // Докл. Адыгской (Черкесской) Междунар. Акад., 2013. Т. 15, № 2. С. 64-67.

27. Толстов Г. П. Ряды Фуръе. М.: Физматлит, 1960. 390 с.

28. Лаврентьев М. А., Шабат Б. В. Методы теории функиий комплексного переменного. М.: Наука, 1965. 716 с.

29. Псху А. В. Краевая задача для уравнения в частных производных дробного порядка в области с криволинейной границей // Докл. Адъгской (Черкесской) Междунар. Акад. наук, 2014. Т. 6, № 2. С. 58-63.

30. Тихонов А. Н., Самарский А. А. Уравнения математической физики. М.: Наука, 1977. $736 \mathrm{c.}$

31. Кошляков Н. С., Глинер Э. Б., Смирнов М. М. Уравнения в частных производных математической физики. М.: Высш. шк., 1970. 710 с.

32. Лыков А. В. Теория теплопроводности. М.: Высш. шк., 1967. 600 с. 
MSC: 26A33, 35K20, 35R11

\title{
The first boundary-value problem in a rectangular domain for a differential equation with the Bessel operator and the Riemann-Liouville partial derivative
}

\section{(C) F. G. Khushtova}

Institute of Applied Mathematics and Automation of Kabardin-Balkar Scientific Centre of RAS,

89 a, Shortanova st., Nal'chik, 360000, Russian Federation.

\begin{abstract}
The paper is devoted to the first boundary-value problem in a rectangular domain for a differential equation with the singular Bessel operator acting with respect to a spatial variable and the Riemann-Liouville fractional differentiation operator acting with respect to a time variable. An explicit representation of the solution is constructed. The uniqueness of the solution is proved in the class of functions satisfying the Hölder condition with respect to the time variable. When the order of the fractional derivative is equal to unity, and the Bessel operator has no singularity, the studied equation coincides with the heat equation and the obtained results coincide with well-known corresponding classical results.
\end{abstract}

Keywords: fractional diffusion equation, fractional differentiation operator, Bessel operator, cylindrical function, Mittag-Leffler type function, first boundary-value problem.

Received: $18^{\text {th }}$ August, 2020 / Revised: $18^{\text {th }}$ May, $2021 /$

Accepted: $24^{\text {th }}$ May, $2021 /$ First online: $30^{\text {th }}$ June, 2021

Competing interests. I have no competing interests.

Author's Responsibilities. I take full responsibility for submitting the final manuscript for print. I approved the final version of the manuscript.

Funding. This research received no external funding.

Acknowledgments. The author thanks two anonymous referees for their careful reading of the article, valuable suggestions and comments.

\footnotetext{
Research Article International License (http://creativecommons.org/licenses/by/4.0/)

Please cite this article in press as: 2021, vol. 25, no. 2, pp. 241-256. https://doi.org/10.14498/vsgtu1820 (In Russian).

Author's Details:

Fatima G. Khushtova (10) https://orcid.org/0000-0003-4088-3621

Cand. Phys. \& Math. Sci.; Researcher; Dept. of Fractional Calculus;

e-mail: khushtova@yandex.ru
}

(우(i) The content is published under the terms of the Creative Commons Attribution 4.0

Khushtova F. G. The first boundary-value problem in a rectangular domain for a differential equation with the Bessel operator and the Riemann-Liouville partial derivative, Vestn. Samar. Gos. Tekhn. Univ., Ser. Fiz.-Mat. Nauki [J. Samara State Tech. Univ., Ser. Phys. Math. Sci.], 


\section{References}

1. Samko S. G., Kilbas A. A., Marichev O. I. Integraly $i$ proizvodnye drobnogo poriadka $i$ nekotorye ikh prilozheniia [Integrals and Derivatives of Fractional Order and Some of Their Applications]. Minsk, Nauka i tekhnika, 1987, 688 pp. (In Russian)

2. Nakhushev A. M. Drobnoe ischislenie $i$ ego primenenie [Fractional Calculus and Its Applications]. Moscow, Fizmatlit, 2003, 272 pp. (In Russian)

3. Pskhu A. V. Uravneniia v chastnykh proizvodnykh drobnogo poriadka [Partial Differential Equations of Fractional Order]. Moscow, Nauka, 2005, 199 pp. (In Russian)

4. Kipriyanov I. A., Katrakhov V. V., Lyapin V. M. On boundary value problems in domains of general type for singular parabolic systems of equations, Sov. Math., Dokl., 1976, vol.17, no. 6 , pp. $1461-1464$.

5. Kipriyanov I. A. Singuliarnye ellipticheskie kraevye zadachi [Singular Elliptic Boundary Value Problems]. Moscow, Nauka, 1997, 204 pp. (In Russian)

6. Zhitomirskii Ya. I. Cauchy's problem for systems of linear partial differential equations with differential operators of Bessel type, Mat. Sb. (N.S.), 1955, vol.36(78), no. 2, pp. 299-310 (In Russian).

7. Matiichuk M. I. Parabolichni singuliarni kraiovi zadachi [Parabolic Singular Boundary-Value Problems]. Kiev, Institute of Mathematics of the Ukrainian National Academy of Sciences, 1999, 176 pp. (In Ukrainian)

8. Matiichuk M. I. Parabolichni ta eliptichni kraiovi zadachi z osoblivostiami [Parabolic and Elliptic Boundary-Value Problems with Singularities]. Chernivtsi, Prut, 2003, 284 pp. (In Ukrainian)

9. Pskhu A. V. The first boundary-value problem for a fractional diffusion-wave equation in a non-cylindrical domain, Izv. Math., 2017, vol.81, no.6, pp. 1212-1233. https://doi.org/ 10.1070/IM8520.

10. Podlubny I. Fractional Differential Equations. An introduction to fractional derivatives, fractional differential equations, to methods of their solution and some of their applications, Mathematics in Science and Engineering, vol. 198. San Diego, CA, Academic Press, 1999, xxiv +340 pp.

11. Kilbas A. A., Srivastava H. M., Trujillo J. J. Theory and Applications of Fractional Differential Equations, North-Holland Mathematics Studies, vol. 204. Amsterdam, Elsevier, 2006, xv+523 pp. https://doi.org/10.1016/S0304-0208(06)80001-0.

12. Metzler R., Glöckle W. G., Nonnenmacher T. F. Fractional model equation for anomalous diffusion, Physica A. Stat. Mech. Appl., 1994, vol.211, pp. 13-24. https://doi.org/10. 1016/0378-4371(94)90064-7.

13. Metzler R., Klafter J. The random walk's guide to anomalous diffusion: a fractional dynamics approach, Phys. Reports, 2000, vol.339, no.1, pp. 1-77. https://doi.org/ 10.1016/S0370-1573(00)00070-3.

14. Uchaikin V. V. Fractional Derivatives for Physicists and Engineers, vol.1, Background and Theory. Berlin, Springer, 2013, xxi+385 pp. https://doi.org/10.1007/ 978-3-642-33911-0.

15. Khushtova F. G. Cauchy problem for a parabolic equation with Bessel operator and Riemann-Liouville partial derivative, Vestn. Samar. Gos. Tekhn. Univ., Ser. Fiz.-Mat. Nauki [J. Samara State Tech. Univ., Ser. Phys. Math. Sci.], 2016, vol. 20, no. 1, pp. 7484 (In Russian). https://doi.org/10.14498/vsgtu1455.

16. Khushtova F. G. First boundary-value problem in the half-strip for a parabolic-type equation with Bessel operator and Riemann-Liouville derivative, Math. Notes, 2016, vol. 99, no. 6, pp. 916-923. https://doi.org/10.1134/S0001434616050308.

17. Khushtova F. G. The second boundary-value problem in a half-strip for a parabolic-type equation with Bessel operator and Riemann-Liouville partial derivative, Math. Notes, 2018, vol. 103, no. 3, pp. 474-482. https://doi.org/10.1134/S0001434618030136.

18. Khushtova F. G. Dirichlet boundary value problem in half-strip for fractional differential equation with Bessel operator and Riemann-Liouville partial derivative, Ufa Math. J., 2017, vol. 9, no. 4, pp. 114-126. https://doi.org/10.13108/2017-9-4-114. 
19. Khushtova F. G. Second boundary-value problem in a half-strip for equation of parabolic type with the Bessel operator and Riemann-Liouvulle derivative, Russian Math. (Iz. VUZ), 2017, vol. 61, no. 7, pp. 73-82. https://doi.org/10.3103/S1066369X17070106.

20. Lebedev N. N. Spetsial'nye funktsii i ikh prilozheniia [Special Functions and Their Applications]. Moscow, Fizmatlit, 1963, 359 pp. (In Russian)

21. Kuznetsov D. S. Spetsial'nye funktsii [Special Functions]. Moscow, Vyssh. shk., 1962, 248 pp. (In Russian)

22. Dzhrbashyan M. M. Integral'nye preobrazovaniia $i$ predstavleniia funktsii $v$ kompleksnoi oblasti [Integral Transforms and Representations of Functions in the Complex Domain]. Moscow, Nauka, 1966, 672 pp. (In Russian)

23. Prudnikov A. P., Brychkov Yu. A., Marichev O. I. Integraly i riady [Integrals and Series], vol. 2, Spetsial'nye funktsii [Special Functions]. Moscow, Fizmatlit, 2003, 664 pp. (In Russian)

24. Stanković B. Inversion et invariantes de la transformation généralisée de Hankel, Acad. Serbe Sci., Publ. Inst. Math., 1955, vol. 8, pp. 37-52 (In French).

25. Pskhu A. V. Integral transformation with Wright's function in the kernel, Dokl. Adygskoi (Cherkes.) Mezhdunar. Akad. Nauk, 2002, vol. 6, no. 1, pp. 35-47 (In Russian).

26. Pskhu A. V. On inversion of the Stanković integral transformation, Dokl. Adygskoi (Cherkes.) Mezhdunar. Akad. Nauk, 2013, vol.15, no. 2, pp. 64-67 (In Russian).

27. Tolstov G. P. Riady Fur'e [Fourier Series]. Moscow, Fizmatlit, 1960, 390 pp. (In Russian)

28. Lavrent'ev M. A., Shabat B. V. Metody teorii funktsii kompleksnogo peremennogo [Methods of the Theory of Functions of Many Complex Variables]. Moscow, Nauka, 1965, 716 pp. (In Russian)

29. Pskhu A. V. A boundary-value problem for the partial differential equation of fractional order in a domain with curvilinear boundary, Dokl. Adygskoi (Cherkes.) Mezhdunar. Akad. Nauk, 2014, vol. 6, no. 2, pp. 58-63 (In Russian).

30. Tikhonov A. N., Samarskii A. A. Uravneniia matematicheskoi fiziki [Equations of Mathematical Physics]. Moscow, Nauka, 1977, 736 pp. (In Russian)

31. Koshlyakov N. S., Gliner E. B., Smirnov M. M. Uravneniia v chastnykh proizvodnykh matematicheskoi fiziki [Partial Differential Equations of Mathematical Physics]. Moscow, Vyssh. shk., 1970, 710 pp. (In Russian)

32. Lykov A. V. Teoriia teploprovodnosti [Theory of Heat Conduction]. Moscow, Vyssh. shk., 1967, 600 pp. (In Russian) 